\title{
Conservation of Energy Momentum and the Metric of a Gravitational or Electromagnetic Plane Wave Pulse
}

\author{
Karl De Paepe ${ }^{1}$ \\ ${ }^{1}$ P.O. Box 1174, Virden, Manitoba, R0M 2C0, Canada \\ Correspondence: Karl De Paepe, P.O. Box 1174, Virden, Manitoba R0M 2C0, Canada. E-mail: \\ k.depaepe@utoronto.ca
}

Received: April 2, 2013 Accepted: May 14, 2013 Online Published: July 15, 2013

doi:10.5539/apr.v5n4p77 URL: http://dx.doi.org/10.5539/apr.v5n4p77

\begin{abstract}
We use conservation of energy and momentum to show the metric of a gravitational or electromagnetic plane wave pulse is flat.
\end{abstract}

Keywords: plane wave pulse, Lorentz transformation, metric, conservation of energy and momentum

\section{Introduction}

A class of Lorentz covariant theories of gravitation that includes general relativity was shown not to satisfy conservation of energy (De Paepe, 2012). There we considered a photon moving along a fixed line towards a particle on the line. In the present article we will consider a gravitational or electromagnetic plane wave pulse incident on an atom that emits a photon. There will be an exchange of energy and momentum between the pulse and the photon. We will require energy and momentum are conserved. This restriction will limit the form of the metric of the pulse.

Consider a gravitational or electromagnetic plane wave pulse moving in the direction of the positive $x$ axis with symmetric total energy-momentum tensor $\tau^{\mu \nu}(t-x)$ having components

$$
\tau^{00}=\tau^{01}=\tau^{11}, \tau^{02}=\tau^{12}=\tau^{22}=\tau^{03}=\tau^{13}=\tau^{23}=\tau^{33}=0
$$

such that $\tau^{\mu \nu}(t-x)=0$ for $x>t$ and $\int_{-\infty}^{\infty} \tau^{00}(u) d u<\infty$. Let the components of the metric be a functions of only $t-x$ and have $g_{\mu \nu}(t-x)=\eta_{\mu \nu}$ for $x>t$. For $x \leq t$ there is no additional restriction on the metric other than it must be the metric of a gravitational or electromagnetic plane wave pulse.

\section{Lorentz Transformations}

We will now construct a Lorentz transformation that will leave the components of (1) unchanged (De Paepe, 2009). Let $\Lambda(\theta)$ be the Lorentz transformation that is a composition of a rotation by $\theta$ about the $z$ axis, a boost by $\frac{2 \cos \theta}{1+(\cos \theta)^{2}}$ in the direction of the rotated $x$ axis, followed by a rotation by $\theta-\pi$ about the $z$ axis. We have $\Lambda(\theta)$ is

$$
\begin{gathered}
t^{\prime}=-t\left[1-2(\csc \theta)^{2}\right]-2 x(\cot \theta)^{2}-2 y \cot \theta \\
x^{\prime}=2 t(\cot \theta)^{2}+x\left[3-2(\csc \theta)^{2}\right]-2 y \cot \theta \\
y^{\prime}=-2 t \cot \theta+2 x \cot \theta+y, z^{\prime}=z
\end{gathered}
$$

Now $\Lambda(\theta)$ satisfies $t^{\prime}-x^{\prime}=t-x$. Also $\Lambda(\theta)$ transforms a test particle with speed one in the direction of the positive $x$ axis to a test particle with speed one in the direction of the $x^{\prime}$ axis.

The total energy-momentum tensor $\tau^{\mu \nu}$ and contravariant form of the metric $g^{\mu \nu}$ transform under Lorentz transformations as

$$
\tau^{\prime \mu \nu}=\frac{\partial x^{\prime \mu}}{\partial x^{\alpha}} \frac{\partial x^{\prime \nu}}{\partial x^{\beta}} \tau^{\alpha \beta} \quad g^{\prime \mu \nu}=\frac{\partial x^{\prime \mu}}{\partial x^{\alpha}} \frac{\partial x^{\prime \nu}}{\partial x^{\beta}} g^{\alpha \beta}
$$

So by (1) and (3) for $\Lambda(\theta)$

$$
\tau^{\prime \mu \nu}\left(t^{\prime}-x^{\prime}\right)=\tau^{\mu \nu}(t-x) ; \int_{-\infty}^{\infty} \tau^{\prime 00}\left(u^{\prime}\right) d u^{\prime}=\int_{-\infty}^{\infty} \tau^{00}(u) d u<\infty
$$


and by (3) for $\Lambda(\theta)$

$$
\begin{gathered}
g^{\prime 00}=\frac{4\left(g^{00}-2 g^{01}+g^{11}\right)}{(\sin \theta)^{4}}+\frac{8\left(-g^{02}+g^{12}\right) \cos \theta}{(\sin \theta)^{3}}+\frac{-4\left(g^{00}-3 g^{01}+2 g^{11}-g^{22}\right)}{(\sin \theta)^{2}} \\
+\frac{4\left(g^{02}-2 g^{12}\right) \cos \theta}{\sin \theta}+g^{00}-4 g^{01}+4 g^{11}-4 g^{22}
\end{gathered}
$$

Since $g^{\mu \nu}$ is a function of only $t-x$ we have for $\Lambda(\theta)$ that $g^{\prime \mu \nu}$ is a function of only $t^{\prime}-x^{\prime}$.

Consider a frame of reference with coordinates $\hat{t}, \hat{x}, \hat{y}, \hat{z}$ related to the coordinates $t, x, y, z$ by the Lorentz transformation

$$
\hat{t}=\frac{t-v x}{\sqrt{1-v^{2}}} \quad \hat{x}=\frac{x-v t}{\sqrt{1-v^{2}}} \quad \hat{y}=y \quad \hat{z}=z
$$

Consequently by (1), (3) with primes replaced by hats, and (6)

$$
\hat{\tau}^{\mu v}(\hat{t}-\hat{x})=\frac{\partial \hat{x}^{\mu}}{\partial x^{\alpha}} \frac{\partial \hat{x}^{v}}{\partial x^{\beta}} \tau^{\alpha \beta}=\frac{1-v}{1+v} \tau^{\mu v}(t-x)
$$

and

$$
\int_{-\infty}^{\infty} \hat{\tau}^{\mu v}(\hat{u}) d \hat{u}=\sqrt{\frac{1-v}{1+v}} \int_{-\infty}^{\infty} \tau^{\mu v}(u) d u<\infty
$$

and by (3) with primes replaced by hats and (6)

$$
\hat{g}^{00}=\frac{\partial \hat{x}^{0}}{\partial x^{\alpha}} \frac{\partial \hat{x}^{0}}{\partial x^{\beta}} g^{\alpha \beta}=\frac{g^{00}-2 v g^{01}+v^{2} g^{11}}{1-v^{2}}
$$

Also $\hat{g}^{00}$ will be function of only $\hat{t}-\hat{x}$.

\section{Conservation of Energy}

Let $\mathcal{F}^{\prime}$ be the frame of reference with the coordinates $t^{\prime}, x^{\prime}, y^{\prime}, z^{\prime}$ of section 2 . In this section we will use conservation of energy to show the function $\left|g^{\prime 00}\right|$ of $\theta$ and $t^{\prime}, x^{\prime}, y^{\prime}, z^{\prime}$ has a maximum.

Let $\overline{\mathcal{F}}$ be a free falling frame of reference with coordinates $\bar{t}, \bar{x}, \bar{y}, \bar{z}$ and with metric $\eta_{\mu \nu}$ at the origin. Let there be a atom $A$ in an excited state that is at rest at the origin with respect to $\overline{\mathcal{F}}$. After some time let $A$ emit a photon $\gamma$ of energy $E$ with respect to $\overline{\mathcal{F}}$. With respect to $\overline{\mathcal{F}}$ let $\bar{p}^{\mu}$ be the components of the energy-momentum four-vector of $\gamma$. We have $\bar{p}^{0}=E$ and $\left(\bar{p}^{0}\right)^{2}=\left(\bar{p}^{1}\right)^{2}+\left(\bar{p}^{2}\right)^{2}+\left(\bar{p}^{3}\right)^{2}$. With respect to $\mathcal{F}$, let $p^{\prime \mu}$ be the components of the energy-momentum four-vector of $\gamma$ at the time of emission. We have

$$
p^{\prime 0}=\frac{\partial x^{\prime 0}}{\partial \bar{x}^{\alpha}} \bar{p}^{\alpha}=\frac{\partial x^{\prime 0}}{\partial \bar{x}^{0}} \bar{p}^{0}+\frac{\partial x^{\prime 0}}{\partial \bar{x}^{1}} \bar{p}^{1}+\frac{\partial x^{\prime 0}}{\partial \bar{x}^{2}} \bar{p}^{2}+\frac{\partial x^{\prime 0}}{\partial \bar{x}^{3}} \bar{p}^{3}
$$

With respect to $\mathcal{F}$ ' at some time let $A$ be at rest at a point in advance of the pulse. In advance of the pulse the system is inertial so if $\gamma$ is emitted before the pulse reaches $A$ the energy of $\gamma$ at the time of its emission will be $E$ with respect to $\mathcal{F}$ '. After some time the pulse will be incident on $A$. By (4) and conservation of energy the amount of energy that $A$ and hence $\gamma$ can gain from the gravitational field of the pulse is limited so the amount $p^{\prime 0}$ can increase from $E$ is limited. Consequently the function $p^{\prime 0}$ of $\theta, t^{\prime}, x^{\prime}, y^{\prime}, z^{\prime}$, and $\bar{p}^{0}, \bar{p}^{1}, \bar{p}^{2}, \bar{p}^{3}$ where $\left(\bar{p}^{1}\right)^{2}+\left(\bar{p}^{2}\right)^{2}+\left(\bar{p}^{3}\right)^{2}=\left(\bar{p}^{0}\right)^{2}=E^{2}$ has a maximum. Note for each $\theta$ we are starting with $A$ at rest in advance of the pulse. Now $p^{\prime 0}$ will be less than or equal to the maximum and $\gamma$ can be emitted in any direction so we can vary $\theta, t^{\prime}, x^{\prime}, y^{\prime}, z^{\prime}$, and vary $\left(\bar{p}^{1}, \bar{p}^{2}, \bar{p}^{3}\right)$ but keep $\left(\bar{p}^{2}\right)^{2}+\left(\bar{p}^{3}\right)^{2}=\left(\bar{p}^{0}\right)^{2}=E^{2}$ to conclude from (10) that the functions $\left(\frac{\partial x^{\prime}}{\partial \bar{x}^{\alpha}}\right)^{2}$ of $\theta$ and $t^{\prime}, x^{\prime}, y^{\prime}, z^{\prime}$ have maxima. Since

$$
g^{\prime 00}=\frac{\partial x^{\prime 0}}{\partial x^{\alpha}} \frac{\partial x^{\prime 0}}{\partial x^{\beta}} \eta^{\alpha \beta}=-\left(\frac{\partial x^{\prime 0}}{\partial \bar{x}^{0}}\right)^{2}+\left(\frac{\partial x^{\prime 0}}{\partial \bar{x}^{1}}\right)^{2}+\left(\frac{\partial x^{\prime 0}}{\partial \bar{x}^{2}}\right)^{2}+\left(\frac{\partial x^{\prime 0}}{\partial \bar{x}^{3}}\right)^{2}
$$

we have the function $\left|g^{\prime 00}\right|$ of $\theta$ and $t^{\prime}, x^{\prime}, y^{\prime}, z^{\prime}$ has a maximum.

\section{Form of Metric}

Since the function $\left|g^{\prime 00}\right|$ of $\theta$ and $t^{\prime}, x^{\prime}, y^{\prime}, z^{\prime}$ has a maximum we must have by (5) that 


$$
\begin{gathered}
g^{00}-2 g^{01}+g^{11}=0, \quad-g^{02}+g^{12}=0, \quad g^{00}-3 g^{01}+2 g^{11}-g^{22}=0, \\
g^{02}-2 g^{12}=0
\end{gathered}
$$

Interchange $y$ and $z$ in the transformation $\Lambda(\theta)$. Using a similar argument

$$
-g^{03}+g^{13}=0, \quad g^{00}-3 g^{01}+2 g^{11}-g^{33}=0, \quad g^{03}-2 g^{13}=0
$$

from which we can conclude $g^{22}=g^{33}$. A rotation about the $x$ axis leaves $\tau^{\mu v}$ unchanged. Make a rotation about the $x$ axis and using a similar argument as before gives $g^{23}=0$. Consequently by (12), (13), and $g^{23}=0$ we can conclude that conservation of energy implies the metric is of form

$$
d s^{2}=L(t-x)\left[-d t^{2}+d x^{2}+d y^{2}+d z^{2}\right]+W(t-x)[d t-d x]^{2}
$$

\section{Conservation of Energy and Momentum}

In this section we use conservation of energy and momentum to show $L=1$. Let $\hat{p}^{\mu}$ be the components of the energy-momentum four-vector of $\gamma$ with respect to the frame of reference of section 2 with coordinates $\hat{t}, \hat{x}, \hat{y}, \hat{z}$. We have

$$
\hat{p}^{0}=\frac{\partial \hat{x}^{0}}{\partial \bar{x}^{\alpha}} \bar{p}^{\alpha}=\frac{\partial \hat{x}^{0}}{\partial \bar{x}^{0}} \bar{p}^{0}+\frac{\partial \hat{x}^{0}}{\partial \bar{x}^{1}} \bar{p}^{1}+\frac{\partial \hat{x}^{0}}{\partial \bar{x}^{2}} \bar{p}^{2}+\frac{\partial \hat{x}^{0}}{\partial \bar{x}^{3}} \bar{p}^{3}
$$

By (8) the amount of energy and momentum of the gravitational field in any cylinder with axis parallel to the $\hat{x}$ axis goes to zero as $v \rightarrow 1$. By conservation of energy and momentum the amount of energy $A$ and hence $\gamma$ can gain or lose from the gravitational field of the pulse goes to zero as $v \rightarrow 1$. Consequently $\hat{p}^{0} \rightarrow E$ uniformly as $v \rightarrow 1$ independent of direction of emission of $\gamma$. We must have by (15) that $\left(\frac{\partial \hat{x}^{0}}{\partial \bar{x}^{\alpha}}\right)^{2} \rightarrow \delta_{\alpha}^{0}$ uniformly as $v \rightarrow 1$. By

$$
\hat{g}^{00}=\frac{\partial \hat{x}^{0}}{\partial x^{\alpha}} \frac{\partial \hat{x}^{0}}{\partial x^{\beta}} \eta^{\alpha \beta}=-\left(\frac{\partial \hat{x}^{0}}{\partial \bar{x}^{0}}\right)^{2}+\left(\frac{\partial \hat{x}^{0}}{\partial \bar{x}^{1}}\right)^{2}+\left(\frac{\partial \hat{x}^{0}}{\partial \bar{x}^{2}}\right)^{2}+\left(\frac{\partial \hat{x}^{0}}{\partial \bar{x}^{3}}\right)^{2}
$$

We have $\hat{g}^{00} \rightarrow-1$ uniformly as $v \rightarrow 1$. By (9), (14), and the fact that $\hat{g}^{00} \rightarrow-1$ uniformly as $v \rightarrow 1$ we can conclude $L=1$.

\section{Conclusion}

The curvature tensor (Weinberg, 1972) of (14) with $L=1$ is zero. The metric is then flat. For a metric and a total energy-momentum tensor that are covariant with respect to Lorentz transformations we showed conservation of energy and momentum implies the metric of a gravitational or electromagnetic plane wave pulse is flat.

\section{References}

De Paepe, K. (2010). Gravitation and Linear Transformation Paradoxes. Physics Essays, 23, 489. http://dx.doi.org/10.4006/1.3462928

De Paepe, K. (2012). Conservation of Energy and Lorentz Covariant Gravitation. Applied Physics Research, 4(4), 115. http://dx.doi.org/10.5539/apr.v4n4p115

Weinberg, S. (1972). Gravitation and Cosmology. New York: John Wiley \& Sons.

\section{Copyrights}

Copyright for this article is retained by the author(s), with first publication rights granted to the journal.

This is an open-access article distributed under the terms and conditions of the Creative Commons Attribution license (http://creativecommons.org/licenses/by/3.0/). 